Splinter Session: X-ray observations of massive stars 


\title{
Stellar Winds in Massive X-ray Binaries
}

\author{
Peter Kretschmar ${ }^{1}$, Silvia Martínez-Núñez ${ }^{2}$, Enrico Bozzo ${ }^{3}$,
} Lidia M. Oskinova ${ }^{4}$, Joachim Puls ${ }^{5}$, Lara Sidoli ${ }^{6}$, Jon Olof Sundqvist ${ }^{7}$, Pere Blay $^{8}$, Maurizio Falanga ${ }^{9}$, Felix Fürst ${ }^{1}$, Angel Gímenez-García ${ }^{10}$, Ingo Kreykenbohm ${ }^{11}$, Matthias Kühnel ${ }^{11}$, Andreas Sander ${ }^{4}$, José Miguel Torrejón ${ }^{10}$, Jörn Wilms ${ }^{11}$, Philipp Podsiadlowski ${ }^{12}$ and Antonios Manousakis ${ }^{13,14}$

${ }^{1}$ European Space Astronomy Centre (ESA/ESAC), Science Operations Department P.O. Box 78, E-28691, Villanueva de la Cañada, Madrid, Spain email: peter.kretschmar@esa.int

${ }^{2}$ Instituto de Física de Cantabria (CSIC-Universidad de Cantabria) E-39005, Santander, Spain

${ }^{3}$ ISDC, University of Geneva, Chemin d'Ecogia 16, Versoix, 1290, Switzerland

${ }^{4}$ Institut für Physik und Astronomie, Universität Potsdam,

Karl-Liebknecht-Str. 24/25, D-14476 Potsdam, Germany

${ }^{5}$ Universitätssternwarte der Ludwig-Maximilians-Universität München, Scheinerstrasse 1, 81679, München, Germany

${ }^{6} \mathrm{INAF}$, Istituto di Astrofisica Spaziale e Fisica Cosmica - Milano, via E. Bassini 15, I-20133 Milano, Italy

${ }^{7}$ Instituut voor Sterrenkunde, KU Leuven, Celestijnenlaan 200D, 3001 Leuven, Belgium

${ }^{8}$ Nordic Optical Telescope - IAC, P.O. Box 474, E-38700, Santa Cruz de La Palma Santa Cruz de Tenerife, Spain

${ }^{9}$ International Space Science Institute (ISSI), Hallerstrasse 6, CH-3012 Bern, Switzerland

${ }^{10}$ Instituto Universitario de Física Aplicada a las Ciencias y las Tecnologías,

University of Alicante, P.O. Box 99, E03080 Alicante, Spain

${ }^{11}$ Dr. Karl Remeis-Observatory \& ECAP, Universität Erlangen-Nürnberg, Sternwartstr. 7, D-96049 Bamberg, Germany

${ }^{12}$ Department of Astronomy, Oxford University, Oxford OX1 3RH, United Kingdom

${ }^{13}$ Centrum Astronomiczne im. M. Kopernika, Bartycka 18, 00-716, Warszawa, Poland

${ }^{14}$ Department of Physics, Sultan Qaboos University, 123 Muscat, Oman

\begin{abstract}
Strong winds from massive stars are a topic of interest to a wide range of astrophysical fields. In High-Mass X-ray Binaries the presence of an accreting compact object on the one side allows to infer wind parameters from studies of the varying properties of the emitted X-rays; but on the other side the accretor's gravity and ionizing radiation can strongly influence the wind flow. Based on a collaborative effort of astronomers both from the stellar wind and the X-ray community, this presentation attempts to review our current state of knowledge and indicate avenues for future progress.
\end{abstract}

Keywords. stars: winds, outflows; supergiants; X-rays: binaries; accretion

\section{Structures in winds from massive stars}

Winds from massive stars are attributed to radiative line-driving, see, e.g., Puls, Vink $\&$ Najarro (2008) for a review. Although the standard theory of line-driven winds assumes a stable, time-independent and homogeneous wind, both theoretical considerations and observational features at different wavelengths clearly indicate that the winds of massive stars are not smooth and isotropic, but structured. 
Small-scale structures are explained by reverse shocks in the wind, which are caused by a very strong, intrinsic instability in line-drive winds (LDI), already noted by Lucy \& Solomon (1970). Numerical hydrodynamical modelling, e.g., by Feldmeier (1995) or Sundqvist \& Owocki (2013) finds that the wind plasma becomes compressed into spatially narrow "clumps" separated by large regions of rarefied gas. The characteristic length scale for these structures is the Sobolev length; for typical hot supergiants this leads to an order of magnitude estimate of $10^{18} \mathrm{~g}$ for typical clump masses and a few $R_{\odot}$ for their extent. See, e.g., Oskinova, Feldmeier \& Kretschmar (2012) for specific predictions.

Large-scale structures in winds from massive stars are mainly inferred from the socalled Discrete Absorption Components (DACs), observed in most O- and early B-star winds (Howarth \& Prinja 1989) and in late B-supergiants (Bates \& Gilheany 1990). A widely held candidate mechanism for these structures are Co-rotating Interaction Regions (Mullan 1984,1986), well studied in the solar wind. Another candidate are Rotational Modulations (RMs), as reported, e.g., by Massa et al. (1995). The density contrasts for these larger structures are rather low (factors of at most a few), but they may contain large overall masses, e.g., $10^{21-22} \mathrm{~g}$ for similar assumptions as above.

\section{Wind-accreting High-Mass X-ray Binaries}

In High-Mass X-ray Binaries (HMXB) a compact object, mostly a neutron star, sometimes a black hole or a white dwarf accretes, matter from its companion and produces copious X-ray radiation. For a typical neutron star $L_{X} \approx 0.1 \dot{M} c^{2}$ for a mass accretion rate $\dot{M}$. There are several mechanisms to fuel the X-ray source, e.g., Roche-Lobe overflow, or from the disk around a Be star - neither discussed further here - but also accretion from the massive star wind. This last mechanism is present in two sub-groups: Classical Supergiant X-ray Binaries (SGXB) tend to be mostly persistent sources with erratic variations in flux. The more recently identified sub-group of Supergiant Fast X-ray Transients (SFXTs) has similar system parameters (where known), but remains mostly in a low luminosity state with brief outbursts and much larger flux variations. For a recent overview of different HXMB in our Galaxy see Walter et al. (2015).

\section{X-ray absorption and fluorescence}

A conceptually straightforward method to infer clumps or larger structures in stellar winds is to measure the attenuation of the X-ray flux, i.e., the variations in the measured absorbing column which in HMXB usually is in the range $N_{\mathrm{H}} \sim 10^{21-24} \mathrm{~cm}^{-2}$. The main caveat is that this requires a good knowledge of the unabsorbed spectral continuum in order to minimise the degeneracy between spectral slope and absorption. An implicit issue is also that accreting X-ray sources are intrinsically variable and thus care has to be taken when comparing different observations. To obtain detailed observational results on wind structures, very extensive campaigns are required, like that reported in Grinberg et al. (2015) and previous publications for Cyg X-1. Large scale structures can also be traced in some cases with the lower time resolution of X-ray monitor data as, e.g., Malacaria et al. (2016) have demonstrated.

Another diagnostic is from X-ray fluorescence lines which will stem mostly from emission nearby to the compact object at most a few $R_{\odot}$ from the X-ray source. The line parameters can yield information on distribution, velocities and ionisation of the reprocessing material as described, e.g., in Giménez-García et al. (2015) and references therein. 


\section{Tracing accreted mass}

As explained above, the X-ray luminosity of an accreting compact object is a direct measure of the current mass accretion rate. Assuming direct infall of matter, the X-ray source would then be a "local probe" of structures in the wind traversed by it. This approach has been used by various authors to explain flares and low-flux or "off" states in HMXB, e.g., by Ducci et al. (2009) or Fürst et al. (2010). But the estimates for clump masses from such studies have sometimes been $2-3$ order of magnitudes larger than those from hydrodynamical simulations of stellar winds.

A closer look at accretion physics also shows that direct infall of captured matter is not necessarily taking place. According to Oskinova, Feldmeier \& Kretschmar (2012), this would also imply orders of magnitude higher variability in many systems than observed. Different studies in recent years discuss, e.g., the possibility of settling envelopes around the compact objects, depending on conditions (Shakura et al. 2012). Another possibility is Chaotic Cold Accretion with complex accretion flows and condensation to filaments and cool clumps, as Gaspari, Temi \& Brighenti (2017) have put forward as a model for AGNs, noting that the findings may also apply to X-ray binaries. In the common case of an accreting neutron star with a strong magnetic field, the interaction with the magnetosphere will lead to additional complications, including possible inhibition of accretion as detailed, e.g., in Bozzo et al. (2008).

\section{Feedback on wind flow}

The presence of the X-ray emitting compact object evidently also influences the wind flow, sometimes quite dramatically so. The gravitational pull focusses the stellar wind in the orbital plane. The bow shock of the compact object moving through the dense wind can create an "accretion wake" following the compact object in its orbit. Also, the intense $\mathrm{X}$-ray emission of bright sources creates a large Strömgren sphere in which the wind is photoionised and the wind acceleration can be slowed or even cut off. These effects have been discussed in quite some detail already by Blondin et al. (e.g., 1990); for recent simulations of these effects see, e.g., Manousakis \& Walter (2015) or Cechura, Vrtilek \& Hadrava (2015). But so far, these feedback models have been based on smooth winds, while models including LDI and clumpy winds have usually not included an accretor and X-ray feedback.

\section{Ongoing efforts and Outlook}

The authors of this contribution and other colleagues have met at the International Space Science Institute (ISSI) Bern for meetings in 2013 \& 2014 and a differently structured follow-up group is meeting again in 2016 \& 2017 in order to discuss the open questions and possible avenues forward.

Among the findings of the first series of meetings are: (1) serious discrepancies in clump sizes and density contrasts used in the literature; (2) systematically lower wind velocities (factor 2-5) in HMXB than those derived for single stars; (3) CIRs should be stable over several orbits, but this is not reflected in HMXB studies of orbital variation; (4) the different behaviour of classical SGXBs and SFXTs remains an open question with no simple explanation. These findings and other results have been published in a detailed review by Martínez-Núnez et al. (2017). The ongoing meetings aim to reduce some of the uncertainties recognised in the first set, as well as include more modelling efforts for wind structure and accretion, and also discuss the impact of these findings for population synthesis studies. 
For the future, we hope to arrive at models combining intrinsically clumpy winds with the effects from Xray feedback, including a realistic picture of time varying accretion and X-ray emission. Systematic multi-wavelength observations via coordinated campaigns with space and ground instruments are required to follow variations on time scales of days or faster. The arrival of fast, sensitive optical spectrographs on ground allows to study some wind variations on time scales of seconds. In space, the advent of X-ray calorimeters will open a new era of X-ray line diagnostics. Until that time, further deep, dedicated observations with the existing grating instruments could still shed light on many questions.

\section{References}

Bozzo, E., Falanga, M., \& Stella, L., 2008, ApJ, 683, 1031

Bates, B. \& Gilheany, S., 1990, MNRAS, 243,320

Blondin, J. M., Kallman, T. R., Fryxell, B. A., \& Taam, R. E., 1990, ApJ, 356, 591

Čechura, J., Vrtilek, S. D., \& Hadrava, P., 2015, MNRAS, 450, 2410

Ducci, L., Sidoli, L., Mereghetti, S., Paizis, A., \& Romano, P. 2009, MNRAS, 398, 2152

Feldmeier, A., 1995, A\&SA, 299, 523

Fürst, F., Kreykenbohm, I., Pottschmidt, K., Wilms, J., Hanke, M., Rothschild, R. E., Kretschmar, P., Schulz, N. S., Huenemoerder, D. P., Klochkov, D., \& Staubert, R. 2010, $A \& A, 519, \mathrm{~A} 37$

Gaspari, M., Temi, P., \& Brighenti, F., 2017, MNRAS, 466, 677

Giménez-García, A., Torrejón, J. M., Eikmann, W., Martínez-Núñez, S., Oskinova, L. M., RodesRoca, J. J., \& Bernabéu, G., 2015, A\& A, 576, A108

Grinberg, V., Leutenegger, M. A., Hell, N., Pottschmidt, K., Böck, M., García, J. A., Hanke, M., Nowak, M. A., Sundqvist, J. O., Townsend, R. H. D., \& Wilms, J., 2015, A\&̈A, 576, A117

Howarth, I. D. \& Prinja, R. K., 1989, ApJS, 69, 527

Lucy, L. B. \& Solomon, P. M., 1970, ApJ, 159, 879

Malacaria, C., Mihara, T., Santangelo, A., Makishima, K., Matsuoka, M., Morii, M., \& Sugizaki, M., 2016, $A \& A$, 588, A100

Manousakis, A. \& Walter, R., 2015, A\& A, 575, A58

Martínez-Núñez, S., Kretschmar, P., Bozzo, E., Oskinova, L. M., Puls, J., Sidoli, L., Sundqvist, J. O., Blay, P., Falanga, M., Fürst, F., Gímenez-García, A., Kreykenbohm, I., Kühnel, M., Sander, A., Torrejón, J. M., \& Wilms, J., 2017, Space Sci. Rev., doi:10.1007/s11214-017-0340-1

Massa, D., Fullerton, A. W., Nichols, J. S., Owocki, S. P., Prinja, R. K., \& 28 co-authors, 1995, ApJL, 452, L53

Mullan, D. J., 1984, ApJ, 283, 303

Mullan, D. J., 1986, A\&ऽA, 165, 157

Oskinova, L. M., Feldmeier, A., \& Kretschmar, P., 2012, MNRAS, 421, 2820

Puls, J., Vink, J. S., \& Najarro, F., 2008, A\&AR, 16, 209

Shakura, N., Postnov, K., Kochetkova, A., \& Hjalmarsdotter, L., 2012, MNRAS, 420, 216

Sundqvist, J. O. \& Owocki, S. P., MNRAS, 428, 1837

Walter, R., Lutovinov, A. A., Bozzo, E., \& Tsygankov, S. S., 2015, A\&AR, 23, 2 